MODELING. IDENTIFICATION AND CONTROL, 2002, vOL. 23, NO. 2, 77-92

doi:10.4173/mic.2002.2.1

\title{
Multi-phase flow modeling applied to metallurgical processes*
}

\author{
STEIN TORE JOHANSEN†
}

Keywords: Multi-phase, multi-scale, flow modeling, metallurgy, dispersed systems, interface tracking, wetting

Multiphase flow models have been improved significantly during the last two decades. Together with the development of more advanced numerical techniques and faster and cheaper computers we now see that computational fluid dynamics (CFD) becomes a powerful tool in predicting the performance of complex industrial processes. In particular the processes faced by the metallurgical industries may serve as examples of such complexity.

In the metallurgical processes transport phenomena take place at a number of different length scales that co-exists in the flow. Heat, mass and momentum exchange, turbulence, reaction kinetics and electromagnetic fields are some of the phenomena, which must be dealt with. Typically, metallurgical applications may include flows with co-existing phases of solids, gas and liquid, which may have internal dispersions of droplets, particles and bubbles. In addition each phase may consist of many different species. Often, the researcher must choose with care the mix of numerical techniques and modeling concepts which, together with the appropriate physics and limited computer power, can bring forward a successful CFD model for a metallurgical process.

This paper will mainly discuss work done by our group at SINTEF Materials Technology. The presented multiphase flow applications range from dispersed to separated flows and include the effects of free surface flows and wetting phenomena.

By using an appropriate mix of techniques the larger scale models may be built on sub models which again can make up the hierarchical structure of a CFD model for an entire process or process unit. The models at each level of such a hierarchical structure may be developed based on more detailed CFD models.

\section{Introduction}

Multiphase flow phenomena are frequently observed in the various metallurgical processes. In all process steps, starting from breaking of ore, processing of minerals, transportation and storage of minerals, feeding furnaces and in the pyro-metallurgical or electrochemical process we find that multiphase flow often phenomena play a dominating role. After the raw metal has been produced the metal is tapped, alloyed to the required composition, slag is separated from metal, solid inclusions are removed, metal is refined by gas injection or bubbling and finally the metal is solidified. Even the latter steps consist of complex multiphase flow phenomena. The complexity of these processes can in some situations be extreme due to electromagnetic fields. high temperatures and heterogeneous chemical reactions.

Situations that involve simultaneously both dispersed flows and interface dynamics are for example found in aluminum reduction cells with anodic bubble formation and metal-bath interfacial waves, in the hearth of blast furnaces and electric arc furnaces and in metal refining units where gas bubble injection and entrainment of air and slag are well known phenomena.

*An early version of this article was presented at the SIMS 2001 conference. October 8th9th, Porsgrunn, Norway.

†SINTEF Materials Technology, Department of Flow Technology, Trondheim, Norway. http://www.sintef.no/flowtech. Email: Stein.T.Johansen@sintef.no 
At present there are no models that can deal with such combined effects and this is a good challenge for further development. However, modeling techniques may be combined sequentially. This was done by Laux et al. (2000), who used a free surface model to compute the entrainment of gas from a tapping jet plunging into a 305 ton ladle. By combining the entrainment rate, predicted from the surface dynamics model, with a two fluid dispersed model, modifications of the flow field due to gas entrainment could be studied.

The paper will show applications of dispersed flow models and demonstrate how free interface dynamics can be used to study processes like metal tapping, filling of metal into moulds and breakup of falling jets during granulation of metals and slags.

An ambition with the paper is to demonstrate how we can use Computational Fluid Dynamics (CFD) to simulate phenomena on different scales, and give some ideas about how this can be use to assemble models on a larger but coarser level.

\section{Model concepts}

The modeling concepts used by the Department of Flow Technology at SINTEF Materials Technology are based on the application of Newton's second law to all materials in question. In particular, each fluid is described by the well-known conservation equations for mass and momentum, expressing mass conservation by;

$$
\frac{\partial}{\partial t}\left(\rho_{f}\right)+\frac{\partial}{\partial x_{j}}\left(\rho_{f} U_{j}\right)=0
$$

and fluid momentum conservation by;

$$
\frac{\partial}{\partial t}\left(\rho_{f} U_{i}\right)+\frac{\partial}{\partial x_{j}}\left(\rho_{f} U_{j} U_{i}\right)=-\frac{\partial}{\partial x_{i}} p+\frac{\partial}{\partial x_{j}} \tau_{j i}+\rho_{f} g_{i}
$$

where $\rho_{f}$ is fluid density, $U_{f}$ is fluid velocity, $p$ is fluid pressure, $g$ is the specific gravity and $\tau_{i j}$ is the fluid stress tensor. When the fluid stress tensor $\tau_{i j}$ is known, such as for a Newtonian fluid, the single-phase flow can be predicted by numerical solution of equations (1) and (2).

\subsection{Dispersed flows}

Extension to multiphase can be done in several ways. Let us first consider a situation when particulates (solid particles, bubbles or droplets) are dispersed in a fluid. The carrier fluid is here described by the Eulerian representation, given by the equations (1) and (2). The particle phase can now be introduced as a continuous field that can penetrate the carrier fluid. Transport equations for the phases appear from volume averages of the fluid and particles in a control volume (Laux, 1998). For a gas-particle system typical transport equations that appear are for mass conservation:

$$
\frac{\partial}{\partial t}\left(\rho_{f} \alpha_{f}\right)+\frac{\partial}{\partial x_{j}}\left(\rho_{f} \alpha_{f} U_{j}\right)=0
$$

and for fluid momentum:

$$
\frac{\partial}{\partial t}\left(\rho_{f} \alpha_{f} U_{i}\right)+\frac{\partial}{\partial x_{j}}\left(\rho_{f} \alpha_{f} U_{j} U_{i}\right)=-\alpha_{f} \frac{\partial}{\partial x_{i}} p+\alpha_{f} \frac{\partial}{\partial x_{i}} \tau_{j i}+\rho_{f} \alpha_{f} g_{i}+\alpha_{p} \beta\left(V_{i}-U_{i}\right)
$$

Here we note that the volume fraction of fluid $\alpha_{f}$ and the inter-phase friction factor $\beta$ (drag term) appear from the volume averages of equations (1) and (2). 
In case of the particle mass balance we obtain:

$$
\frac{\partial}{\partial t}\left(\rho_{p} \alpha_{p}\right)+\frac{\partial}{\partial x_{j}}\left(\rho_{p} \alpha_{p} V_{j}\right)=0
$$

and for particle momentum:

$$
\begin{aligned}
\frac{\partial}{\partial t}\left(\rho_{p} \alpha_{p} V_{i}\right)+\frac{\partial}{\partial x_{j}}\left(\rho_{p} \alpha_{p} V_{j} V_{i}\right)= & -\frac{\partial}{\partial x_{i}} p^{s}+\frac{\partial}{\partial x_{j}} \tau_{j i}^{s}+\rho_{p} \alpha_{p} g_{i}-\alpha_{p} \frac{\partial}{\partial x_{i}} p \\
& +\alpha_{p} \frac{\partial}{\partial x_{j}} \tau_{j i}+\alpha_{p} \beta\left(U_{i}-V_{i}\right)
\end{aligned}
$$

Here $\alpha_{p}$ is the volume fraction of particles. Because of inter-particle collisions and momentum exchange due to collisions both the solids pressure $p^{s}$ and the solid particle internal stress $\tau_{j i}^{s}$ is included in the equations. For more details about the origin of these equations see Laux (1998). It should be noted that in case of interphase mass transfer and more complex interphase momentum exchange mechanisms, these equations must be modified. For many metallurgical applications chemical species must be conserved within each phase. In addition the averaging procedure must be performed for the energy equation in order to yield volume-averaged equations for conservation of enthalpy.

The velocities are now averages from small control volumes and are no longer the instantaneous velocities given in the equations (1) and (2). In a swarm of large particles the fluid velocity close to the particle surface is very different from the volume-averaged velocity. However, the effects of the local variations will in practice only affect the interphase transfer terms such as drag and mass transfer.

An alternative way to include a second phase into a model is by using a Lagrangian description for the dispersed phase. In this case particle trajectories are calculated on a given flow field. The particle position is advanced in time by the momentum equation:

$$
m_{p} \frac{d}{d t} V_{i}=m_{p} g_{i}+k_{i}+f_{\text {ext }, i}
$$

where $V_{i}$ is particle velocity, $m_{p}$ is particle mass, $k_{i}$ is the forces caused by the surrounding fluid and $f_{\text {ext.,i }}$ is the external body forces (not gravity). The particle position $X_{i}$ is calculated from:

$$
\frac{d}{d t} X_{i}=V_{i}
$$

By using the 'Particle Source in Cell' concept due to Crowe et al. (1977) the influence of particles on the fluid's momentum can be calculated. The Lagrangian treatment of particles can be extended to deal with particle-particle interactions by the socalled Discrete Element Method (DEM) (Johansen and Laux, 1999). Sawley and Cleary (1999) used this method to study the particle flow and inter-particle impacts in a high intensity grinding mill. See Figure 1.

\subsection{Separated flows}

When large regions of separated fluids coexist, the methods described above are hard to adopt. In this case it is possible to track the positions of the interfaces with 


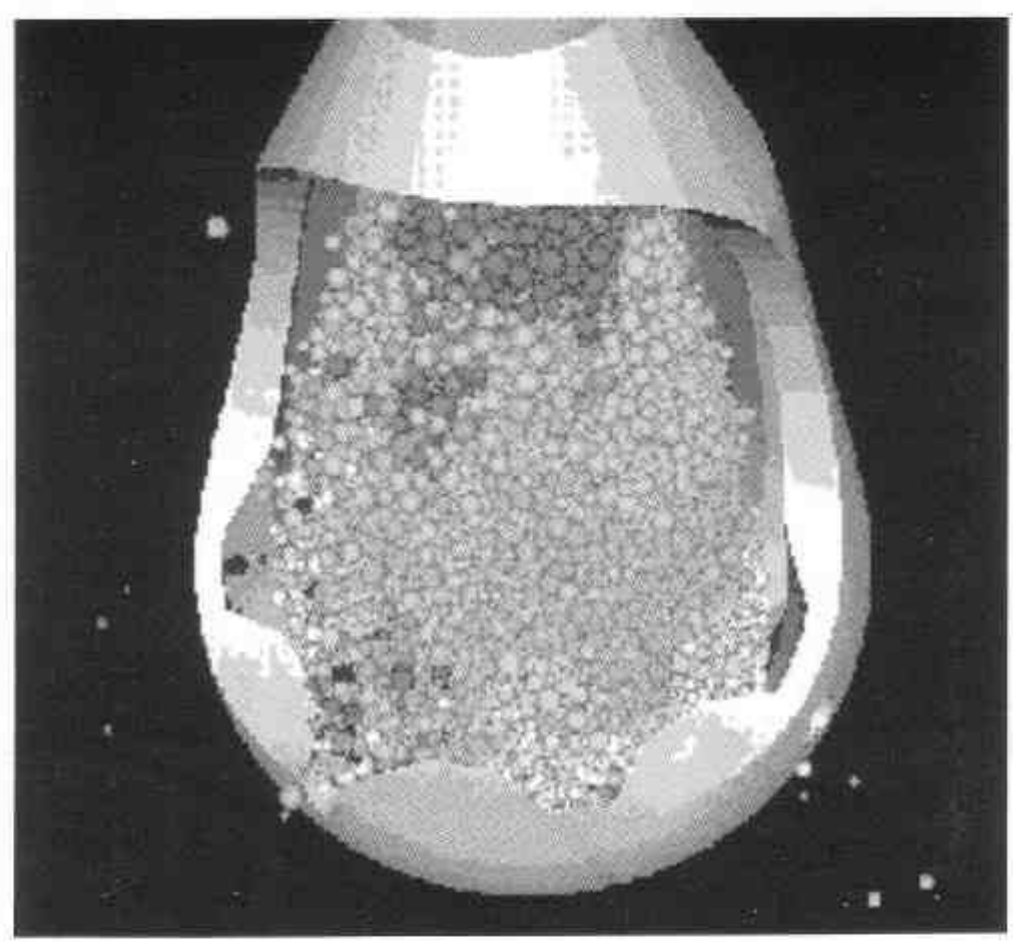

Figure 1. From a simulation of particle milling using the Discrete Element Method (DEM) (Sawley and Cleary, 1999). All individual particles are represented by the method.

appropriate methods and solve a single-phase momentum equation for the entire flow domain (Johansen, 2001). In such models the interface is a transition region where density and viscosity change abruptly over typically one single computational cell. The effects of surface tension and wetting (Johansen, 2001) can be treated using continuous fields, associated with the interface itself. We use the method described in reference (Johansen, 1998) to investigate free surface flow phenomena.

Wetting has recently been added to our separated flow model. At present only three wetting regimes can be reproduced. These regimes are: (i) No wetting, (ii) $90^{\circ}$ wetting angle and (iii) Complete wetting. Numerically these regimes are represented by setting the interface normal vector at the walls. In the case of a gas liquid system with no wetting, (i), it is assumed that there is a microscopic gas film covering the wall when liquid contacts the wall. In the full wetting case, (iii), it is assumed that all walls have a thin film of liquid. The $90^{\circ}$ contact angle is represented by the interface normal vector that always is tangential to the wall.

We investigated the full wetting implementation on an initially cylindrically shaped droplet of water, with equivalent diameter $9.08 \mathrm{~mm}$, that was placed inside a cylinder of $10 \mathrm{~mm}$ of height and $20 \mathrm{~mm}$ of diameter. The grid consisted of 40 cells in both the axial and radial direction.

Predicted interface shapes are shown in the Figures 2 and 3. It is interesting to note the apparent wetting angle observed in Figure 2. The Youngs contact angle imposed on the system is $180^{\circ}$. However, the apparent contact angle is here a result of gravity and surface tension. This tells us that:

(i) The contact angle cannot be read from experiments like Figure 2 without careful analyses.

(ii) Numerical methods that tend to enforce a given contact angle on the interface are bound to fail. This has until recently been a popular method. 


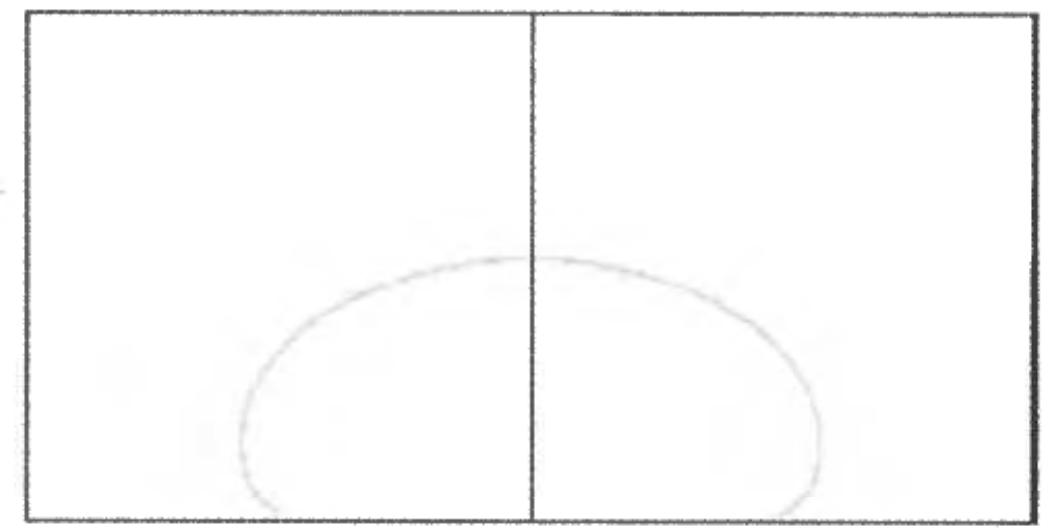

Figure 2. Non wetted walls: Predicted equlibrium shape of $9 \mathrm{~mm}$ diameter water droplet.

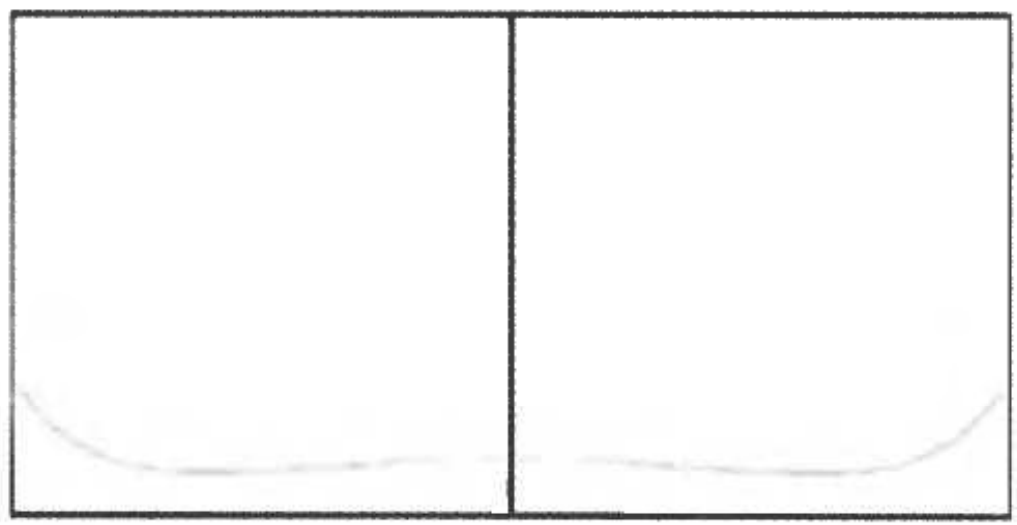

Figure 3. Fully wetted walls: Predicted interface shape of $9 \mathrm{~mm}$ water droplet at time $\mathrm{t}=0.30 \mathrm{sec}$.

\subsection{Turbulent flows}

In most industrial flow situations the flow is turbulent. Therefore the model equations (3) to (6) must be time averaged or ensemble averaged. By using mass weighted ensemble averages for velocity, as demonstrated in Laux (1998), phenomena like turbulent dispersion can be treated rigorously. The turbulence models are multiphase adoptions of the well-known $\mathrm{k}-\varepsilon$ model concept. In the case of dense particle suspensions we need to add model equations for the particle phase turbulent energy and dissipation rate (Laux, 1998).

When Lagrangian methods are applied for the dispersed phase, turbulent dispersion of particles are simulated by imposing random generated turbulent velocities (Johansen, 1990).

In dynamic simulations like free surface flows, turbulence is most often treated by a sub-grid Large Eddy turbulence model, such as the Smagorinski model (Smagorinsky, 1963).

All these models need appropriate boundary conditions in order to achieve a high quality of the predictions. The most successful boundary conditions are the wall laws (Johansen, 1990) which try to express wall fluxes as analytic expressions without solving numerically for the details in the flow boundary layers. 


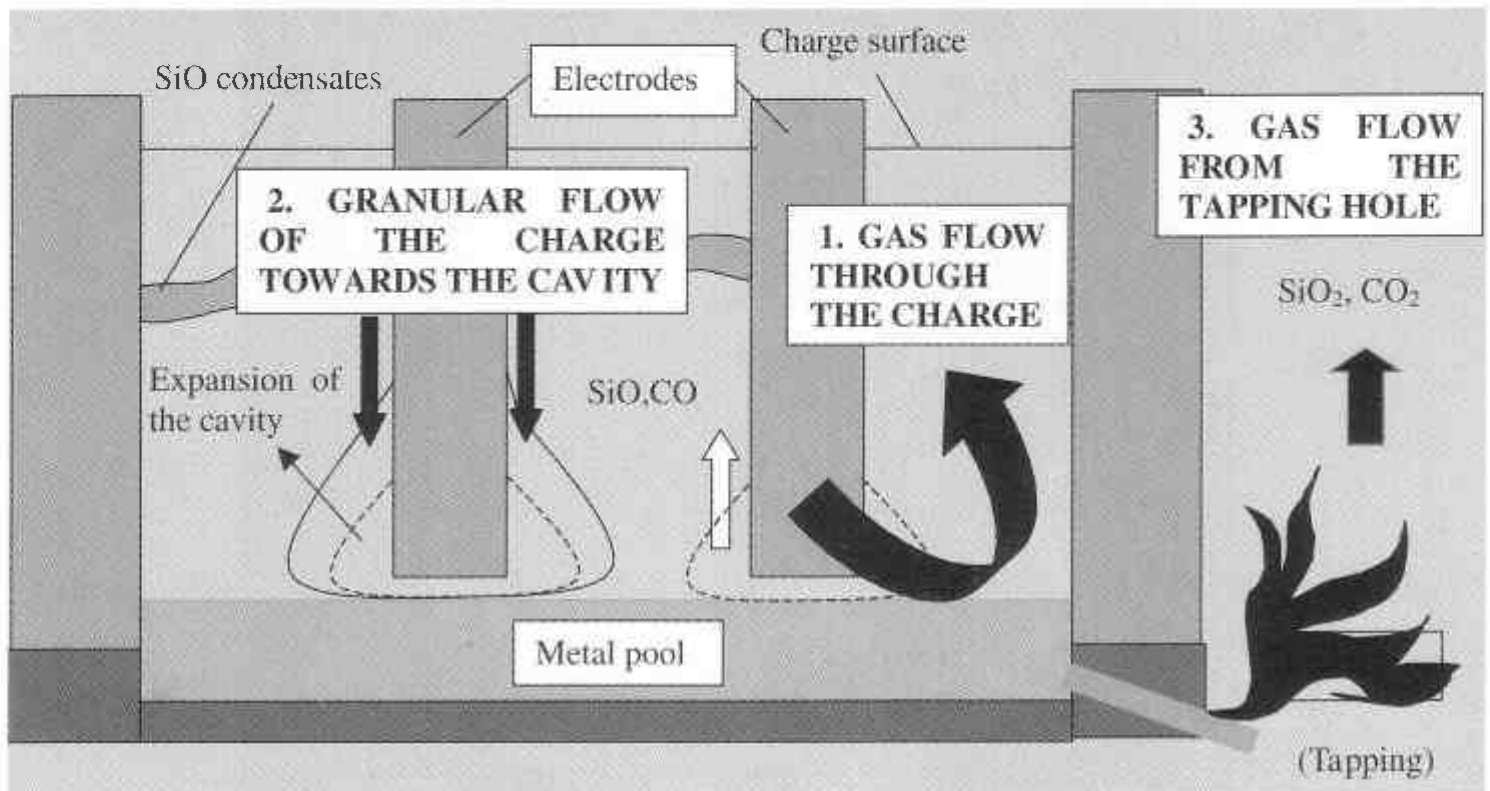

Figure 4. Principal sketch of a Ferro Silicon furnace (Ravary, 2000). The raw materials are fed on the charge surface and flow slow down to the reaction zone (cavity area).

\section{Industrial applications}

In order to deal with the various multiphase flow phenomena related to the metallurgical industry a mixture of techniques may often be an advantage. In order to have a flexible tool to simulate the various phenomena we use the commercial FLUENT $^{\mathrm{TM}}$ Code, which in some situations have been modified in source code or by using User Defined Subroutines (UDF).

\subsection{The hierarchy of scales}

In most metallurgical applications models must treat phenomena at a large number of different scales simultaneously. A typical example is a Silicon furnace, as seen in Figure 4. Here the geometrical scales of the system range from micrometers (pores in carbon materials), to centimeters (quarts and iron pellets), further to meters (electrode diameter) and finally to the full diameter of the furnace that may be typically $10 \mathrm{~m}$. In practical calculations the phenomena that take place on scales below, say $10 \mathrm{~cm}$, must be modeled and cannot be predicted directly.

In order to model these sub-grid phenomena the flow of gas or liquid slag through a packed bed can be studied by single flow simulations as indicated in Figure 5. From this type of simulation the pressure drop can be predicted and correlations regarding the permeability may be obtained for each particular system. Hence, the inter-phase friction factor $\beta$ in equation (4) may be obtained even if experimental data is not available.

We can now use the equations (3) and (4) to predict the flow of gas out from the reaction zone of a Ferro Silicon furnace. A resulting flow field of the mainly vertical gas flow is seen in Figure 6. In this case the bed of solid raw materials is stagnant. The horizontal cross-section is slightly above the tip of the electrodes, as seen in Figure 4. Note that, with the actual permeability for the packed bed of raw materials, the gas flow is concentrated in between the electrodes.

By using the granular flow concept, illustrated by the equations (3)-(6), it is 


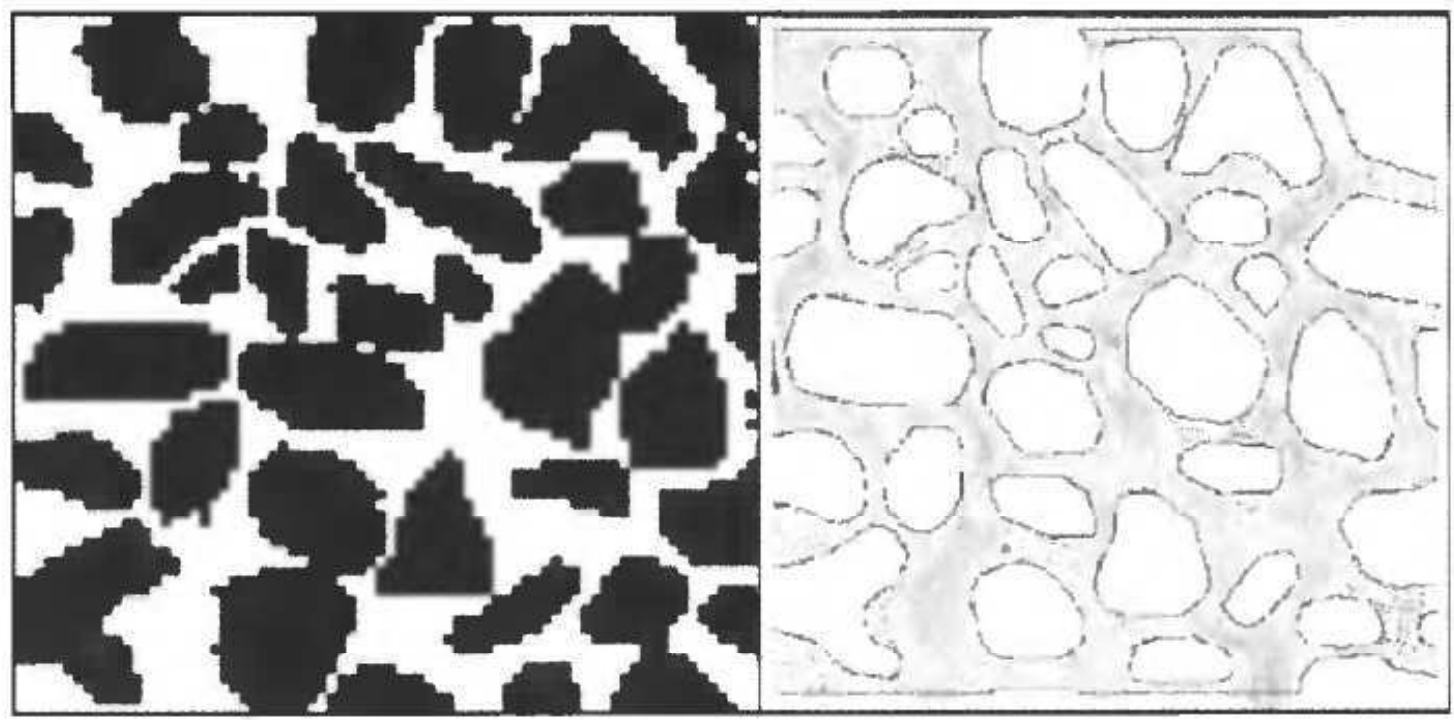

Figure 5. To the left a digitized image of a sample of packed solid raw materials. The right hand picture shows the predicted fluid flow passing through such a bed.

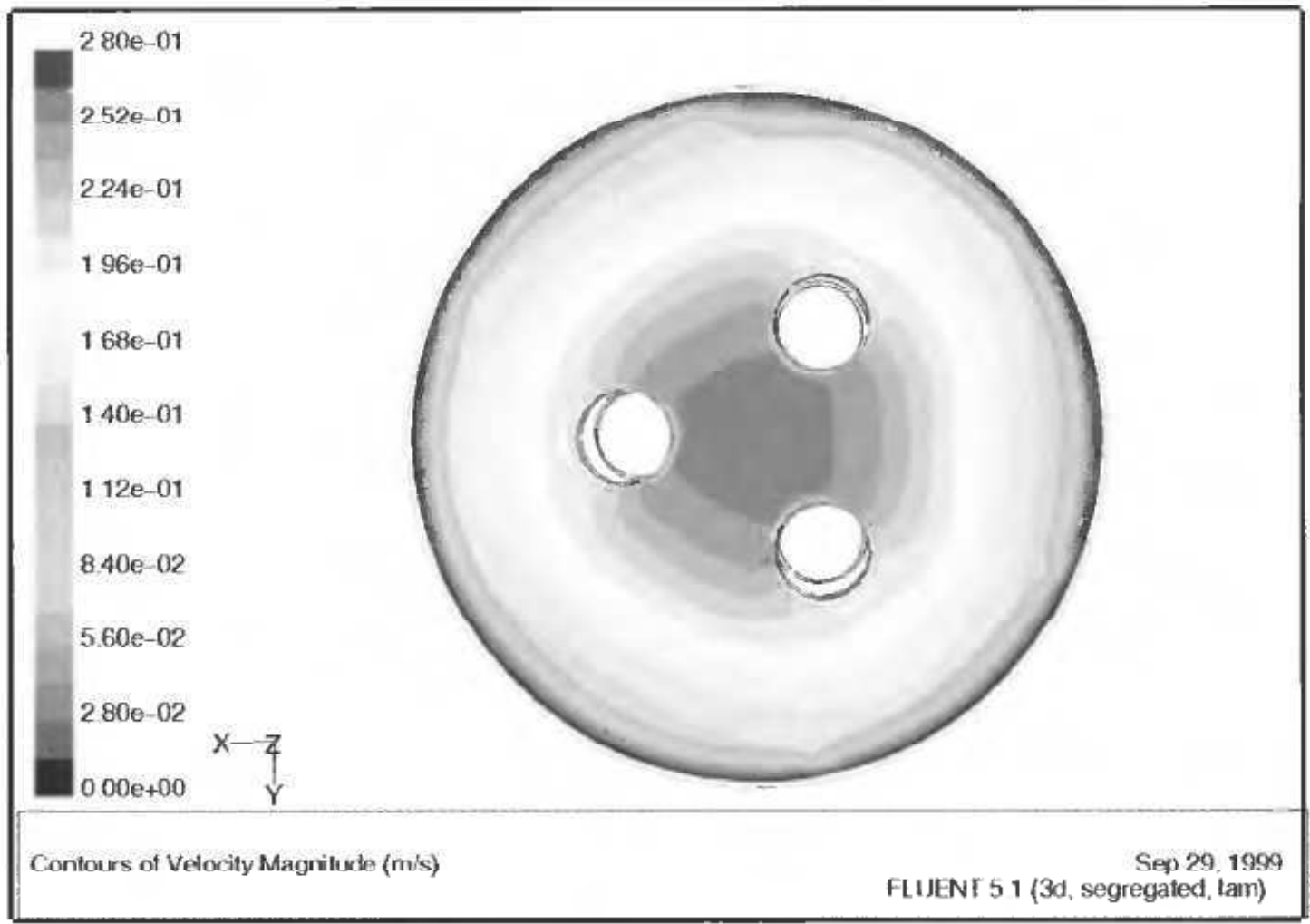

Figure 6. The velocity distribution of CO-gas up from the bottom of a FeSi furnace (Ravary and Laclau, 1999).

possible to study the flow of raw materials and gas released from the process in a two-phase simulation. In Figure 7 we see the prediction of the charge surface and the velocities of raw materials and process gas. In this case the simulation is simplified to a 2D axi-symmetrical geometry with only one electrode involved. It is assumed that there is no shear stress from the raw materials on the electrode. The result is in good qualitative agreement with observation of charge movements during furnace operation (Skei et al., 1998). 

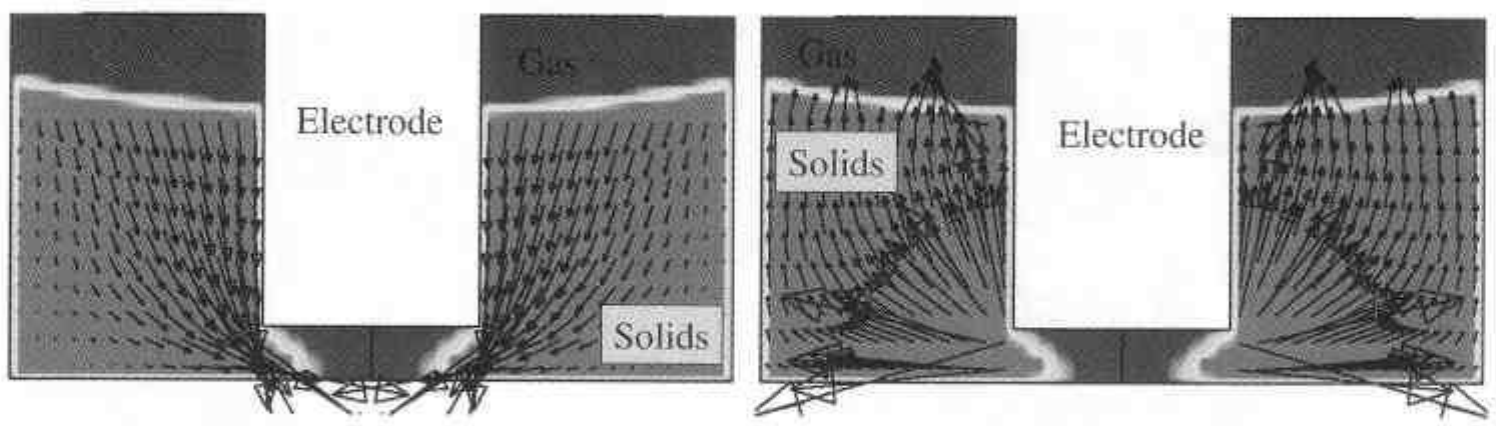

Figure 7. Simulation of flow in FeSi-furnace using the granular flow model (Laux, 1998). In the middle we se the electrode. Initially, in the computation, the bed of particles is flat after charging. Both figures show the volume fraction of solid materials after a given time. To the left we se velocity vectors for the solids. To the right the velocity vectors for the gas is displayed.

\subsection{Tapping phenomena in ferro-alloy furnaces}

As indicated in Figure 4, metal is tapped from holes in the side of these furnaces, typically ranging from 10 to $40 \mathrm{MW}$ of electric power. The pool of metal indicated in Figure 4 may typically contain up to 40 tons of ferro-alloy. In the side lining of the furnace a hole is maintained that is opened at regular intervals. The mouth of this hole is planned to be well below the metal surface. However, immediately after opening, large amounts of combustible SiO-gas often escape from the furnace, mainly because of the large internal pressure caused by the reactions of the process itself. Flames from these 'blow-outs' are a potential risk for the operators and lead to pollution of the working environment. By using a free surface flow model (Johansen, 1998) we can explain the phenomenon in a simple manner.

In Figure 8 a porous region, resembling observed regions of porous sludge, is placed inside the furnace. This porous region is in the model blocking the direct access of metal to the tapping hole. In this case the metal close to the tapping hole

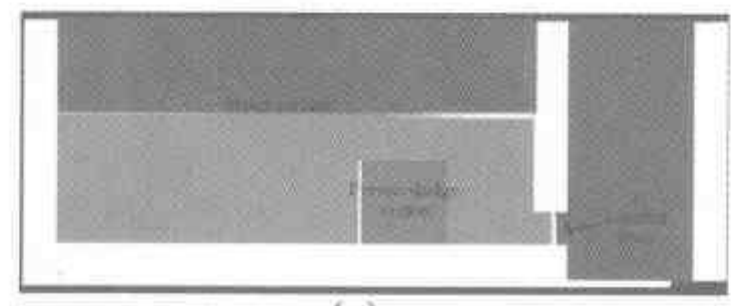

(a)

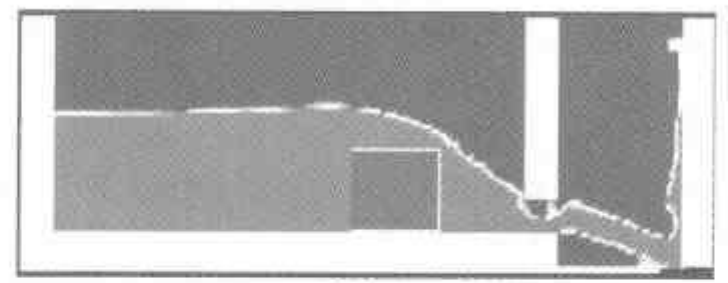

(c)

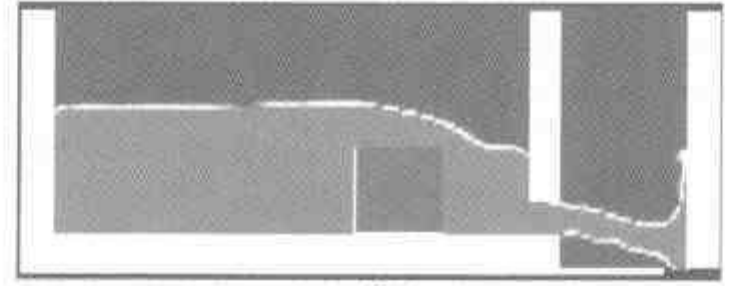

(b)

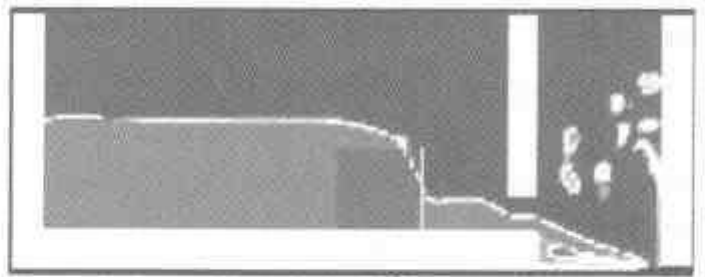

(d)

Figure 8. The figure shows a tapping sequence in a ferro-alloy furnace. The refractory walls are colored grey. Initial metal pad depth is $30 \mathrm{~cm}$. (a) Initial configuration; (b) Tapping is started; (c) First break-through of process gas; and (d) Strongly reduced metal flowrate. 
is rapidly drained out and process gas will escape together with metal during the complete tapping period. In order to improve the situation it is important to find methods to prevent formation of the low permeability sludge region inside the metal pool.

\subsection{Gas entrainment during tapping of steel into ladles}

During tapping of steel from a steel converter to a ladle the fall height of the free steel jet may be more than $10 \mathrm{~m}$, with a typical jet diameter of $10 \mathrm{~cm}$. Two phenomena are of particular interest: (i) nitrogen entrained by the plunging jet into the ladle may deteriorate the steel quality, and (ii) entrained gas may influence the flow pattern in the ladle significantly and thereby effect the dissolution and mixing of alloy elements.

In Figure 9 we see the predicted gas entrainment in a 2D axisymmetric simulation. The flow is not well resolved and the bubbles are numerically diffused by the numerical technique (Johansen, 1998). However, the predicted entrainment flowrates

(a)

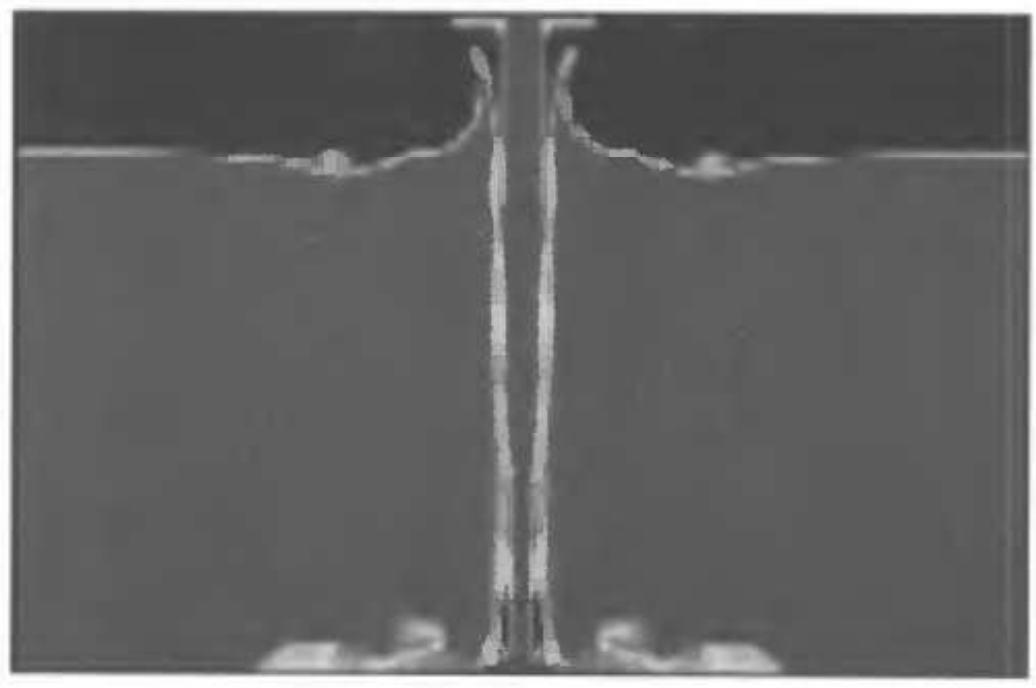

(b)

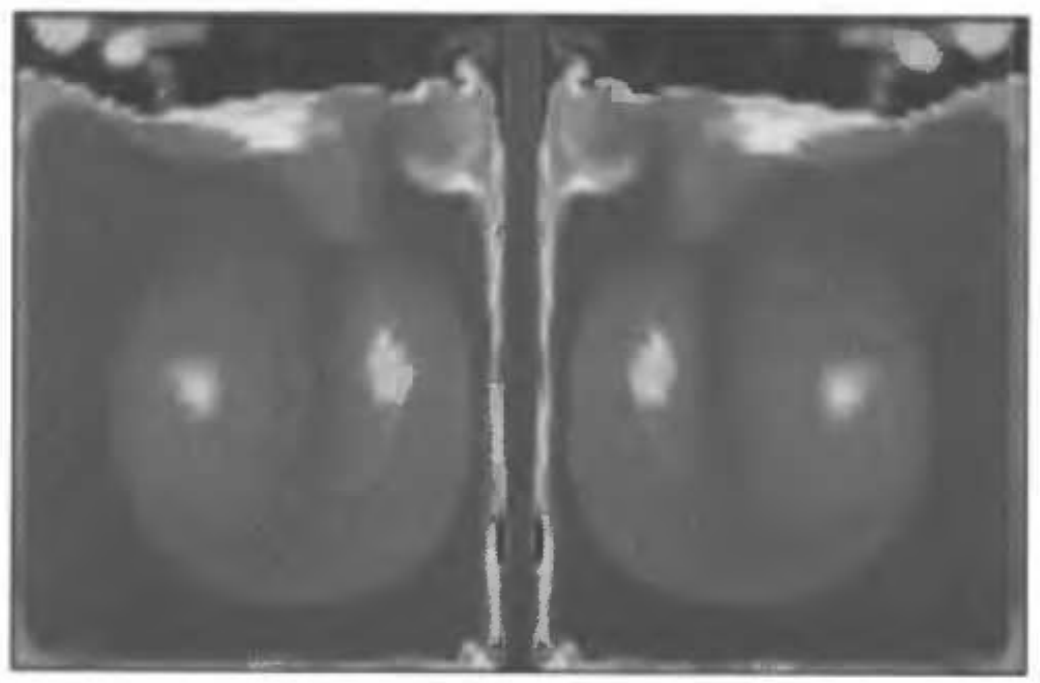

Figure 9. (a) Steel jet, plunging into a ladle at $40 \%$ filling (Laux, 2001). First bubbles arrive at the ladle bottom; (b) Steel jet, plunging into a ladle at $40 \%$ filling (Laux, 2001). Surface entrainment is initiated. 


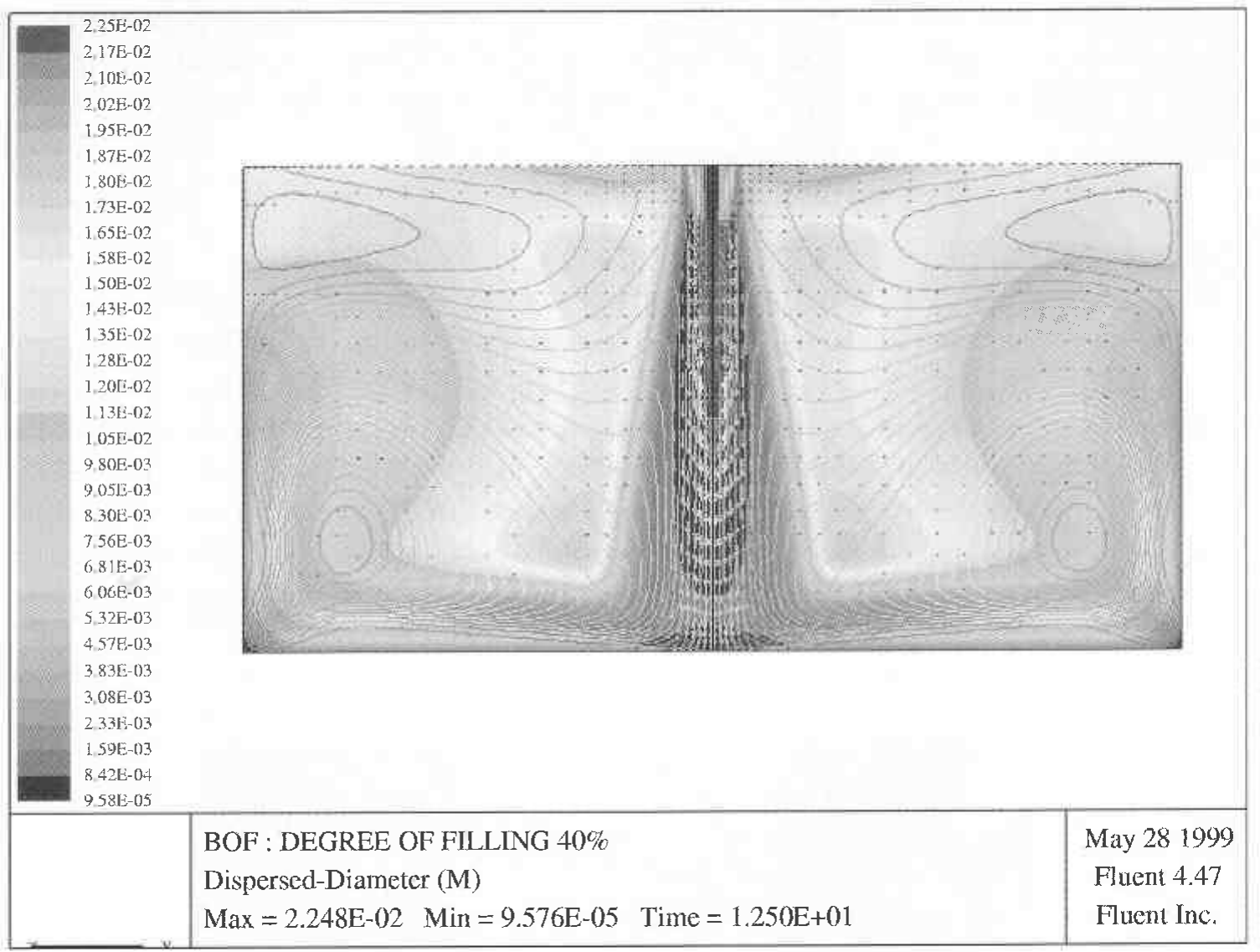

Figure 10. The figure displays the predicted average bubble diameter distribution as well as the flow streamlines (Laux et al., 2001). The modeling technique is the dispersed flow Eulerian description. Note that flow direction is parallel to the streamlines. The blue arrows indicate the plunging jet seen in Figure 9.

are comparable to results from experimental correlations (Laux et al., 2001). In order to predict the effect of gas entrainment on the dissolution rate of alloy materials we use an Eulerian description of the bubble phase (Laux et al., 2001), where the bubble size is predicted from a transport model (Laux and Johansen, 1999). The gas entrainment rate predicted by the free surface model, as seen in Figure 9, is here used as a boundary condition. Using a Lagrangian description of the alloy particles (Berg et al., 1999), the effect of entrained gas on flow pattern and alloy particle dissolution have been studied (Laux et al., 2001).

In Figure 10 we see the predicted bubbles sizes (background color) and the liquid steel streamlines (Laux et al., 2001). Inside the jet region the predicted bubble sizes are typically below $5 \mathrm{~mm}$ in diameter. We see that the tapping jet penetrates to the bottom of the ladle and rises up along the sidewall. However, close to the jet boundary the presence of bubbles supplies sufficient buoyancy to turn the direction of the flow. Accordingly, the entrainment of gas leads to an outward flow in the surface. This surface flow direction is opposite of what is the case when gas entrainment is not present.

\subsection{Phenomena related to metal casting}

During the process of transferring a liquid metal to some solid state, metal must often be filled into some die or mould. During the filling process the evolvement of 


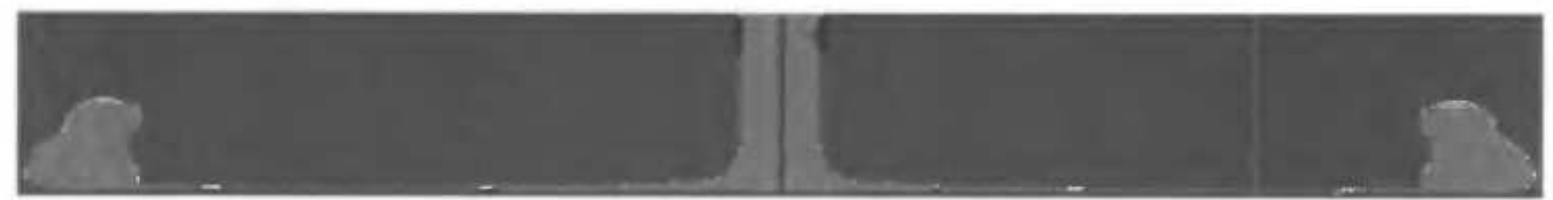

Figure 11. A liquid jet of water spreads on a non-wetted substrate.

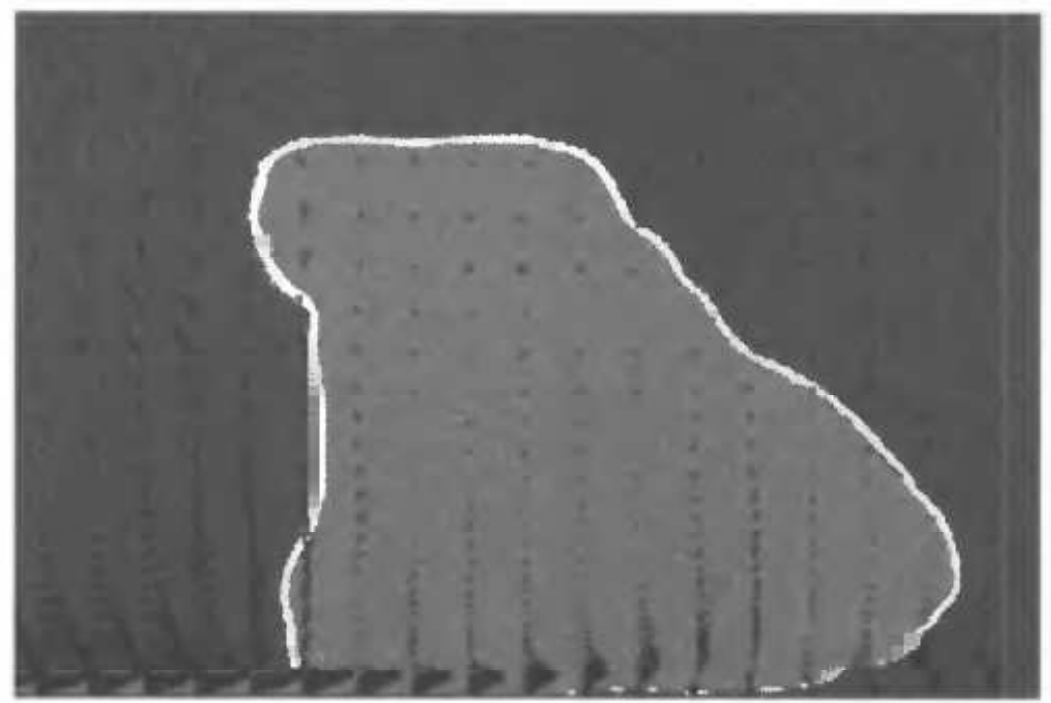

Figure 12. The flow inside the hydraulic jump seen in Figure 11.

gas pockets that may lead to porosities is of great interest. Phenomena related to wetting of the mould are also of primary interest. In Figure 11 we see a liquid jet of water with diameter $4.4 \mathrm{~mm}$ and vertical velocity $3.0 \mathrm{~m} / \mathrm{s}$. The jet enters centrally at the top, hits the mould and spreads radially outwards in a thin film of approximately $200 \mu \mathrm{m}$ of thickness. The height of the domain is $10 \mathrm{~mm}$ and the gas wets the substrate completely. We see that a hydraulic jump is produced, initially due to the surface tension of the water. Figure 12 shows a close up of the flow inside the hydraulic jump. It is interesting to note that the flow separates inside the jump, contrary to current understanding of the phenomenon.

When a liquid metal is poured onto a plate like seen in Figure 11, we have a typical casting situation for some metallurgical products. However, by repeating the simulation above for liquid aluminum, it was found that the metal would not spread like seen in Figure 11. The metal will first collect in a metal balloon until the volume of the balloon is sufficiently large to start spreading. This phenomenon disappears at sufficiently large initial velocities and flow-rates.

Another challenging phenomenon is the breakup of liquid metal jets in water. By pouring liquid metal into water the jet breaks up and forms droplets that solidify as granules. This is today a popular method for casting ferro-alloys.

The granulation of liquid metals is a complex phenomenon as it involves a large number of phenomena. When the liquid metal is pored into water, breakup of the metal jet is initiated, as predicted in Figure 13. At the same time vapor evolves on the metal surface, effecting the heat transfer and solidification, effecting the surface dynamics that controls droplet breakup. In worst cases powerful and damaging explosions may occur that may lead to severe injuries of plant personnel. 

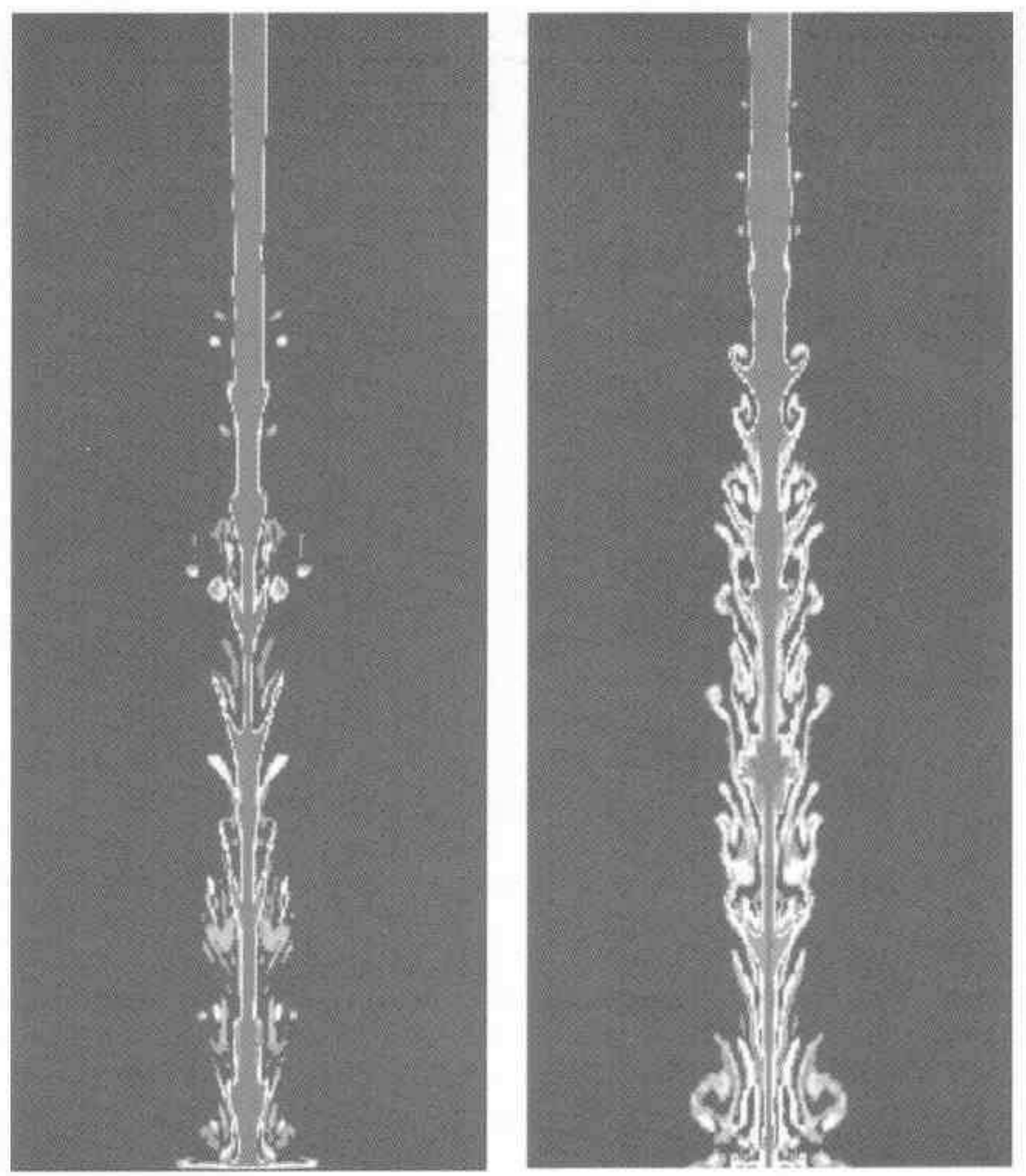

Figure 13. Snapshots of cylinder-symmetrical isothermal predictions of the shape of a jet of liquid metal plunging into water. The initial jet diameter is $16 \mathrm{~mm}$, and the length of the vessel is $500 \mathrm{~mm}$. To the left: Initial velocity is $1.0 \mathrm{~m} / \mathrm{s}$. To the right: Initial jet velocity is $4.0 \mathrm{~m} / \mathrm{s}$.

In the snapshots from the iso-thermal simulations seen in Figure 13 we see that fast jet to the right seems to break up faster than the slower left jet. Both jets are perturbed by the same initial instability wavelength. However, it is evident that the fast jet that experience more shear force from the surrounding liquid is more unstable. Note that the main breakup mechanism is the shedding of toroidal structures away from the jet. These structures form individual droplets by capillary breakup in the circumferential direction. After individual droplet formation these droplets are expected to fall down as 'rain' surrounding the incoming jet. In order to capture these phenomena the simulations must be repeated in full 3D.

\subsection{Effects of wetting during anodic gas release in aluminum electrolyses}

In aluminum electrolyses the process gas developed at the anodes forms bubbles that escape at regular intervals. If the bottom surface of the anodes are slightly 
(a)

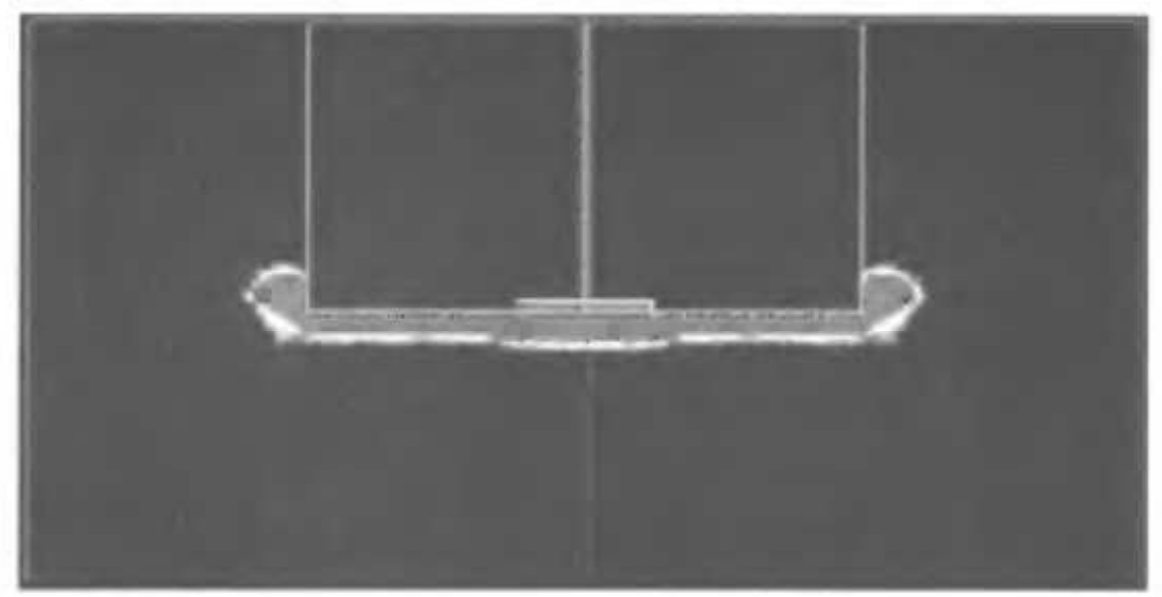

(b)

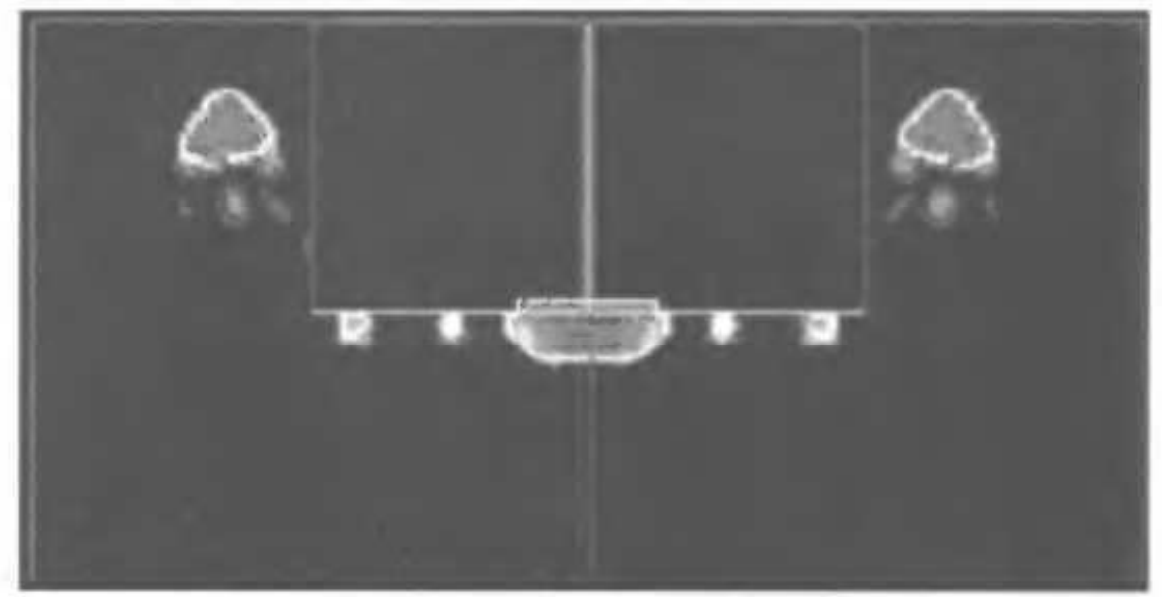

Figure 14. (a) Gas release from wetted anode; (b) Released gas bubble, drifting away from anode surface.

deviating from the horizontal the bubble will slide easily along the anode surface. Such bubbles have been simulated by Rudman (1998). We have used Langrangian techniques to study bubble movement and convection, heat and mass transfer controlled by these bubbles (Solheim et al., 1989; Haarberg et al., 1998). However, it is not known what will happen in industrial operation if the anodes are completely horizontal (Shekar and Evans, 1994). In the cylinder symmetrical simulations shown in Figure 14, the anode is completely wetted by the liquid. The anode diameter is $30 \mathrm{~cm}$ and the liquid is cryolite.

When the bubbles detach from the anode surface most of the gas is drained away from the surface, leaving a surface with good electric contact to the liquid. It is interesting to note that the bubble shapes create sufficient lift to move the bubbles well away from the side of the anode.

However, on the contrary, if the gas wets the anode the behavior is completely changed, as seen in Figure 15. Now the gas sticks to the anode and forms a more or less permanent gas sheet that increases the electrical resistance between anode and liquid considerably. This is in good agreement with the current understanding of the anode effect. During the anode effect the concentration of dissolved alumina becomes sufficiently low to produce poor wetting of the anode, leading to a high electric 
(a)

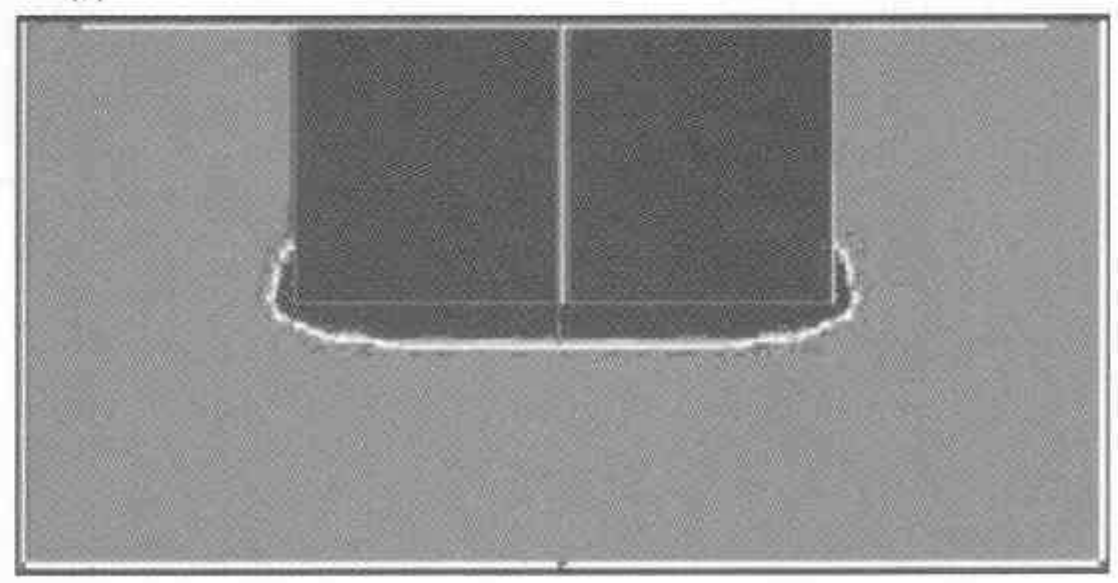

(b)

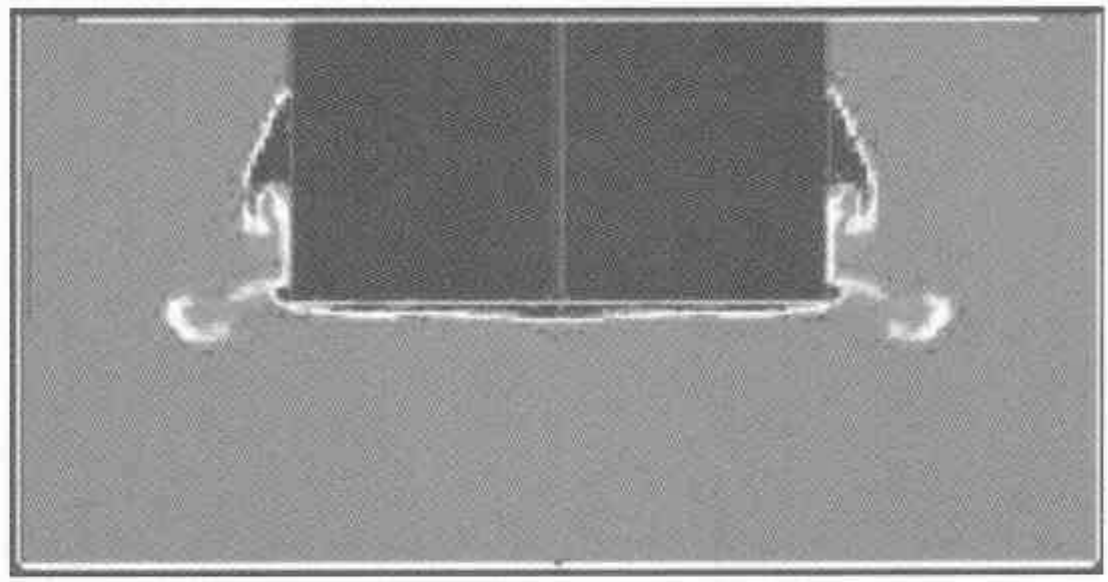

Figure 15. (a) Gas release from non-wetted anode; (b) Released gas bubble, sliding along the surface of the non-wetted anode.

resistance and strong ohmic heating. Note that the bubbles now slide along the anode surface and are not able to detach from the anode.

\section{Conclusions}

By starting out from single phase flow modeling it is possible to build more complex multi-field and multiphase models. These models can be used to study complex metallurgical systems with bubble break-up and fragmentation as well as chemical reactions and phase transitions with heat and mass transfer.

It has further been demonstrated that also free surface modeling techniques can be used to explain a number of industrial problems. The number of possible industrial applications for such techniques is large.

The free surface techniques have a large potential in simulating detailed flow phenomena. Results from such simulations can be used to develop sub-models for studies of phenomena at larger scales. These sub-models will then be used in Eulerian multi-field models that has the potential to describe very complex systems without modeling every detail directly. These macro level models will be statistically averaged, but may even so be a powerful tool in improving and designing industrial processes.

Experimental verifications at all levels are crucial for predictive CFD models. 
Better experimental techniques for validation of CFD models for metallurgical applications will be one important cornerstone in the future development of this field.

\section{Acknowledgement}

The Research Council of Norway is acknowledged for supporting general development in this field under the program CARPET (www.carpet.ntnu.no), project 140527/ 420. My colleague Harald Laux is acknowledged for proofreading the manuscript.

\section{REFERENCES}

Berg, H., Laux, H., Johansen, S. T. and Klevan, O.-S. (1999). Flow pattern and alloy dissolution during tapping of steel furnaces, Ironmaking \& Steelmaking. 26, 127-139.

Crowe, C. T., Sharma, M. P. and Stock, D. E. (1977). The particle source in Cell (PSI-Cell) model for gas-droplet flows, J. Fluids Engr., 99, 235.

HaArberG, T., SOlheim, A. and Johansen, S. T. (1998). Effect of anodic gas release on current efficiency in Hall-Héroult celles, Light Metals, edited by Barry Welch, TMS, pp. 475-481.

JOHANSEN, S. T. (1990). On the modelling of turbulent two-phase flows. Dr.Techn.-thesis. The Norwegian Institute of Technology, Trondheim.

JOHANSEN, S. T. (1998). Large scale simulation of separated multiphase flows, Proceedings of Third International Conference on Multiphase Flows, ICMF '98, Lyon, France, June 8-12, 1998, CD-ROM.

JOHANSEN, S. T. (2001). Multiphase flow modeling of metallurgical flows. Proceedings of the 4th International Conference on Multiphase Flow, ICMF 2001, New Orleans, LA, USA, May 27-June 1, 2001, CD-ROM, Paper 204.

Johansen, S. T. and Laux, H. (1999). Simulations of Granular Materials Flows, Proceedings of RELPOWFLO III, Proceedings of the International Symposium on the Reliable Flow of Particulate Solids, 11-13 August 1999, Telemark College. Porsgrunn, Norway, 1999, 11 pages.

Laux, H. (1998). Modeling of dilute and dense dispersed fluid-particle two-phase flow, Ph.D. thesis 1998:71, Norwegian University of Science and Technology.

LaUX, H. (2001). Private communication.

Laux, H., Bech, K. H., Hellevik, L. R. and Johansen, S. T. (2001). CFD modeling of bubble-driven flow, Proceedings of MekIT '01, Trondheim 3-4 May 2001, pp. 279-300, and is accepted for publication in International Journal of Applied Mechanics in Engineering (IJAME), 17(1).

Laux, H. and Johansen, S. T. (1999). A CFD Analysis of the Air Entrainment Rate Due to a Plunging Steel Jet Combining Mathematical Models for Dispersed and Separated Multiphase Flows, Fluid Flow Phenomena in Metals Processing, edited by N. EL-KadDaH et al.. TMS, 21--30.

LAUX, H. and JOHANSEN, S. T. (2001). Eulerian multiphase modeling of bubbly flow in a gasstirred ladle, Proceedings of the 4th International Conference on Multiphase Flow, ICMF 2001, New Orleans, LA, USA, May 27-June 1, 2001, CD-ROM, Paper 203.

I.AIIX, H., Johansen, S. T., BerG, H and KLEVAN, O-S. (2000). CFD analyses of turbulent flow in ladles and the alloying process during tapping of steel furnaces, Scandinavian Journal of Metallurgy, 29, 71-80.

Laux, H., Johansen, S. T., Berg, H. and Klevan, O.-S. (2001). Gas-induced motion in metallurgical ladles due to gas-entrainment during tapping of steel furnaces, SINTEF Report STF24 A01605.

RaVARY, B. (2000). Beregninger av strømning i chargen og ovnsrom. Presentation at The FFFsymposium, 25-26 Oktober 2000, Kristiansand.

Ravary, B. and LACLAU, J.-C. (1999). Modelling of the charge flow around the electrode, the gas flow in the charge and out of the taphole in FeSi furnaces, SINTEF Report STF24 F99626.

Rudman, M. J. (1998). A volume-tracking method for incompressible multifluid flows with large density variations, Int. J. Numer. Meth. Fluids, 28, 357-378.

Sawley, M. L. and Cleary, P. W. (1999). A parallel discrete element method for industrial granular flow simulations, EPFL Supercomputing Review, Nov., 23-29 
Shekrhar, R. and Evans, J. W. (1994). Physical Modeling Studies of Electrolyte Flow due to Gas Evolution and Some Aspects of Bubble Behavior in Advanced Hall Cells: Part I. Flow in Cells with a Flat Anode, Met. Trans. B, 25B, 333-340.

Skei, A., Tuset, J. KR. and Tvert, H. (1998). Production of High Silicon Alloys, Tapir Forlag, Trondheim.

SMAGORINSKY, J. (1963). General circulation experiments with the primitive equations: Part I. The basic experiment, Mon. Weather Rev., 91, 99-164.

Solheim, A., Johansen, S. T., Rolseth, S. and Thonstad, J. (1989). Gas Driven Flow in HallHeroult Cells, Light Metals, TMS, pp. 245-252. 